\title{
Linear Stability and Nonlinear Analysis of an Extended Optimal Velocity Model Considering the Speed Limit
}

\author{
Guangzhu He, Cuncai Hua \\ Department of Mathematics, Yunnan Normal University, Kunming, China \\ Email: hgzwxm201202@163.com,cuncai-hua@139.com
}

How to cite this paper: He, G.Z. and Hua, C.C. (2020) Linear Stability and Nonlinear Analysis of an Extended Optimal Velocity Model Considering the Speed Limit. Journal of Applied Mathematics and Physics, 8, 507-518.

https://doi.org/10.4236/jamp.2020.83040

Received: February 20, 2020

Accepted: March 14, 2020

Published: March 17, 2020

Copyright $\odot 2020$ by author(s) and Scientific Research Publishing Inc.

This work is licensed under the Creative

Commons Attribution International

License (CC BY 4.0).

http://creativecommons.org/licenses/by/4.0/

\begin{abstract}
In this paper, an extended car-following model is proposed based on an optimal velocity model (OVM), which takes the speed limit into consideration. The model is analyzed by using the linear stability theory and nonlinear analysis method. The linear stability condition shows that the speed limit can enlarge the stable region of traffic flow. By applying the reductive perturbation method, the time-dependent Ginzburg-Landau (TDGL) equation and the modified Korteweg-de Vries (mKdV) equation are derived to describe the traffic flow near the critical point. Furthermore, the relation between TDGL and $\mathrm{mKdV}$ equations is also given. It is clarified that the speed limit is essentially equivalent to the parameter adjusting of the driver's sensitivity.
\end{abstract}

\section{Keywords}

Optimal Velocity Model (OVM), Speed Limit, TDGL Equation, mKdV Equation

\section{Introduction}

With the development of social economy and the increasing number of motor vehicles, traffic jam has become the common bottleneck of urban development. The problem of traffic congestion can be attributed to the stability and solitary waves of traffic flow models. Generally speaking, traffic flow models have been divided into macroscopic models, microscopic models, and mesoscopic models according to the aggregation level. There are many microscopic models in traffic flow, and the most commonly studied model is the car-following model. The car-following model is a microscopic traffic flow model used to describe the behavior of a single driver. In order to study the complex characteristics of traffic 
flow, scholars have proposed various car-following models to understand the formation and transmission mechanism of traffic congestion.

The early research work on the car-following theory was originated from Reuschel [1] and Pipes [2]. Since then, scholars have carried out many related research works. In 1961, Newell [3] proposed a car-following model of velocity-governing equation by assuming that the velocity adjustment of the rear car depends on the optimized velocity of the headway. In 1995, Bando et al. [4] proposed the optimal velocity model (OVM), in which the optimal velocity was determined only by the headway with the forward vehicle. Despite the simplicity and few parameters, the OVM can be used to describe various characteristics of the actual traffic flow, such as stop-and-go phenomenon, the instability of traffic flow and the evolution of traffic jams. In 1998, Helbing and Tilch [5] verified OVM by using empirical data and proposed a generalized force model (GFM), which overcomes the problems of unrealistic deceleration and excessive acceleration of OVM. In 2001, Jiang et al. [6] found that the GFM could not be used well to describe the delay time and the kinematic wave speed at jam density and proposed a full velocity difference model (FVDM) by considering the influence of positive and negative speed differences on the car-following models. In 2008, in order to overcome the high deceleration of FVDM, Ge et al. [7] proposed a two-velocity difference model (TVDM) based on the application of intelligent transportation system (ITS). Recently, some new car-following models were submitted to describe traffic nature more realistically [8] [9] [10] [11] [12]. Undoubtedly, these scholars have made various contributions to the establishment and development of traffic flow. For the above car-following models, there have been many researches focusing on the stability and density waves in the past time.

In the past, more research has focused on the density waves for the car-following models. However, it is still enormous significance to derive thermodynamic theory of traffic flow model and to link traffic meta-stability with spinodal decomposition in first-order phase transition [13]. The TDGL equation can refer to non-equilibrium phase transition [14]. Nagatani [15] firstly proposed the thermodynamic theory of traffic flow, and derived the TDGL equation of two simple lattice hydrodynamic models near the critical point and the thermodynamic potential of traffic flow by applying the reduced perturbation method. So far, there has been little research on deriving TDGL equation from car-following models [16] [17] [18] [19]. Since the TDGL equation of the traffic flow model can be used to represent the thermodynamics theory of jamming transition, it is very important to establish the traffic flow model and derive the corresponding TDGL equation.

In recent years, traffic jams are still intense as the number of cars in big cities increases and road infrastructure becomes more complex. Exploitation of intelligent transport system is a part of the best ways for settling transport problems. Considering the influence of an intelligent transportation system, many researchers have established some car-following models to describe the real nature 
of traffic flow [20] [21] [22] [23] [24]. In fact, intelligent vehicle navigation system can provide service for the vehicles, when the actual speed of the vehicle deviates from the speed limit. If the vehicle overspeeds, it is easy to cause traffic congestion and traffic accidents, and the driver can learn the speed limit information in advance, he can adjust in advance to achieve a stable speed. At this time, the traffic can develop smoothly. In other words, drivers' knowledge of speed limit information in advance plays an important role in stabilizing the traffic flow. However, most of the car-following models have not considered the effect of the speed limit.

In order to reveal the effect of the speed limit on traffic flow, an extended optimal velocity model is introduced by considering the speed limit in this paper. The content of this paper is organized as follows. In Section 2, an extended optimal velocity model with consideration of the speed limit is introduced. In Section 3, the linear stability condition is obtained from the linear stability analysis method. In Section 4, the TDGL equation and its corresponding soliton solution are obtained from the reductive perturbation method. In Section 5, the mKdV equation is derived in the unstable region. In Section 6, conclusions are given.

\section{An Extended OVM with Speed Limit}

In 1995, Bando et al. [4] proposed the OVM based on the analysis of the characteristics of traffic flow. The equation of motion is as follows:

$$
\frac{\mathrm{d}^{2} \Delta x_{n}(t)}{\mathrm{d} t^{2}}=a\left[V\left(\Delta x_{n}(t)\right)-\frac{\mathrm{d} \Delta x_{n}(t)}{\mathrm{d} t}\right]
$$

where $a$ is the driver's sensitivity coefficient, $x_{n}(t)>0$ is the position of the vehicle at time $t, V(\cdot)$ is the optimal velocity function, and $\Delta x_{n}(t)=x_{n+1}(t)-x_{n}(t)$ represents the space headway between the car ahead and the car following at time $t$.

As people make full use of the latest information of the intelligent transportation system, some traffic problems are gradually solved. For example, intelligent vehicle navigation system uses computer and communication technology to provide information relevant to the cars on the road and generate dynamic traffic information, such as the speed limit [25]. It can determine at what appropriate velocity the driver should drive under the current conditions according to the current traffic conditions and signal status. When the actual velocity of the vehicle deviates from the speed limit by the navigation system, the driver will adjust the actual velocity according to the stable speed limit to improve the traffic capacity and relieve traffic congestion. Therefore, we propose the following extended optimal velocity model by considering the speed limit:

$$
\frac{\mathrm{d}^{2} \Delta x_{n}(t)}{\mathrm{d} t^{2}}=a\left[V\left(\Delta x_{n}(t)\right)-\frac{\mathrm{d} \Delta x_{n}(t)}{\mathrm{d} t}\right]+\lambda a\left(V_{l i m}-\frac{\mathrm{d} \Delta x_{n}(t)}{\mathrm{d} t}\right),
$$

where $V_{\text {lim }}$ is the speed limit provided by the navigation system, and $\lambda$ de- 
notes the reactive coefficient related to the speed limit $V_{\text {lim }}$.

We chose the optimal velocity function $V\left(\Delta x_{n}(t)\right)$ as proposing in reference [4].

$$
V\left(\Delta x_{n}(t)\right)=\frac{v_{\max }}{2}\left[\tanh \left(\Delta x_{n}(t)-h_{c}\right)+\tanh \left(h_{c}\right)\right]
$$

where $v_{\max }$ is the maximum velocity of the vehicles, $h_{c}$ is the safety distance of the vehicles. The optimal velocity function $V(\cdot)$ is a function of $\Delta x_{n}(t)$, and it's a monotonically increasing function with an upper bound.

For the convenience of analysis in the following, we discretize Equation (2) with asymmetric forward difference [24], and rewrite it in terms of headway as follows:

$$
\Delta x_{n}(t+2 \tau)=\Delta x_{n}(t+\tau)+\tau\left[V\left(\Delta x_{n+1}(t)\right)-V\left(\Delta x_{n}(t)\right)\right]-\lambda\left[\Delta x_{n}(t+\tau)-\Delta x_{n}(t)\right]
$$

where $\tau$ is time step and $\tau=\frac{1}{a}$.

\section{Linear Stability Analysis}

In this section, we study the extended optimal velocity model of Equation (4) by the method of linear stability analysis.

First, we assume that the initial state is stable, the headway of the vehicle is $h$ and the corresponding optimal velocity is $V(h)$. At this time, the vehicle position of the steady-state traffic flow can be expressed as

$$
x_{n}^{0}(t)=h n+V(h) t, \quad h=L / N,
$$

where $N$ is the number of cars, $L$ is the road length, and $h$ is the average headway. Let $y_{n}(t)$ be a small deviation from the steady-state solution $x_{n}^{0}(t)$, then the perturbed solution is given as

$$
x_{n}(t)=x_{n}^{0}(t)+y_{n}(t)
$$

Substituting (6) into Equation (4), we obtain the following formulation:

$$
\Delta y_{n}(t+2 \tau)=\Delta y_{n}(t+\tau)+\tau V^{\prime}\left[\Delta y_{n+1}(t)-\Delta y_{n}(t)\right]-\lambda\left[\Delta y_{n}(t+\tau)-\Delta y_{n}(t)\right],
$$

where $\Delta y_{n}(t)=y_{n+1}(t)-y_{n}(t)$ and $V^{\prime}(h)=\left.\frac{\mathrm{d} V\left(\Delta x_{n}(t)\right)}{\mathrm{d} t}\right|_{\Delta x_{n}=h}$.

Let $\Delta y_{n}(t)=\exp (i k n+z t)$, Equation (7) can be rewritten as follows:

$$
\mathrm{e}^{2 z \tau}=\mathrm{e}^{z \tau}+\tau V^{\prime}\left(\mathrm{e}^{i k}-1\right)-\lambda\left(\mathrm{e}^{z \tau}-1\right) .
$$

where $V^{\prime}=V^{\prime}(h)$. Since $z \rightarrow 0$ as $i k \rightarrow \infty, z$ can be expressed by a long wave as $z=z_{1}(i k)+z_{2}(i k)^{2}+\cdots$.

Substituting it into (8) and neglecting the higher order items, we obtain the coefficients of the first-and second-order term of $i k$, as follows:

$$
z_{1}=\frac{V^{\prime}(h)}{1+\lambda}, \quad z_{2}=\frac{V^{\prime}(h)}{2(1+\lambda)}-\frac{V^{\prime 2}(h) \tau(3+\lambda)}{2(1+\lambda)^{3}} .
$$


Clearly, if $z_{2}$ is negative, the initial steady uniform flow will become unstable; if $z_{2}$ is positive, the original steady flow state remains unchanged. Thus, the neutral stability condition of an extended optimal velocity model considering the speed limit is obtained as follows:

$$
\tau=\frac{(1+\lambda)^{2}}{V^{\prime}(h)(3+\lambda)} .
$$

We are concerned with $\tau$ relating to $\lambda$, the reactive coefficient relating to $V_{\text {lim }}$. Thus, the uniform traffic flow remains stable if the following condition holds:

$$
\tau<\frac{(1+\lambda)^{2}}{V^{\prime}(h)(3+\lambda)} .
$$

As $\lambda>0$, we have $\frac{1}{3 V^{\prime}(h)}<\frac{(1+\lambda)^{2}}{V^{\prime}(h)(3+\lambda)}$. This shows that the stable region of (11) is larger than that of (12) the OVM (where $\lambda=0$ ) given as follows:

$$
\tau<\frac{1}{3 V^{\prime}(h)} .
$$

From (11), we can see that the parameter $\lambda$ has an important effect on the stabilization of traffic flow. Notice that $\left[\frac{(1+\lambda)^{2}}{3+\lambda}\right]=\frac{(1+\lambda)(5+\lambda)}{(3+\lambda)^{2}}>0, \lambda>0$, at this time, $\frac{(1+\lambda)^{2}}{3+\lambda}$ is increases monotonically with respect to $\lambda$, so it holds that $\frac{1}{3 V^{\prime}(h)}<\frac{(1+\lambda)^{2}}{V^{\prime}(h)(3+\lambda)}, \lambda>0$. As a result, the stability condition (11) is weaker than (12), and the stable region is larger than OVM.

Figure 1 shows the phase diagram in the $(h, a)$-phase where $h$ (meter) is the headway and $a$ (1/second) is sensitivity which corresponds to the inverse of the delay time. It shows the stable neutral lines of (10) with different values of $\lambda$, where $v_{\max }=2 \mathrm{~m} / \mathrm{s}$ is taken as the maximal velocity and $h_{c}=4 \mathrm{~m}$ is the safe distance. In Figure 1, above the neutral stability curve is a stable region, which represents a free phase without traffic congestion, and below the neutral stability curve is an unstable region, which represents the traffic jam phase that evolves backward as the density waves stop-and-go. The peak of each curve represents the critical point $\left(h_{c}, a_{c}\right)$. Figure 1 also shows that as the sensitivity coefficient $\lambda$ to the speed limit increases, the stable region also gradually expands. This shows that if the driver can obtain the speed limit information through the intelligent vehicle navigation system in advance, he can adjust the vehicle speed earlier, effectively enhance the stability of the traffic flow, and avoid traffic congestion.

\section{TDGL Equation}

Now we use the long-wavelength modes to derive the TDGL equation for describing the pedestrian flow on a coarse-grained scale [26]. The long-wavelength 


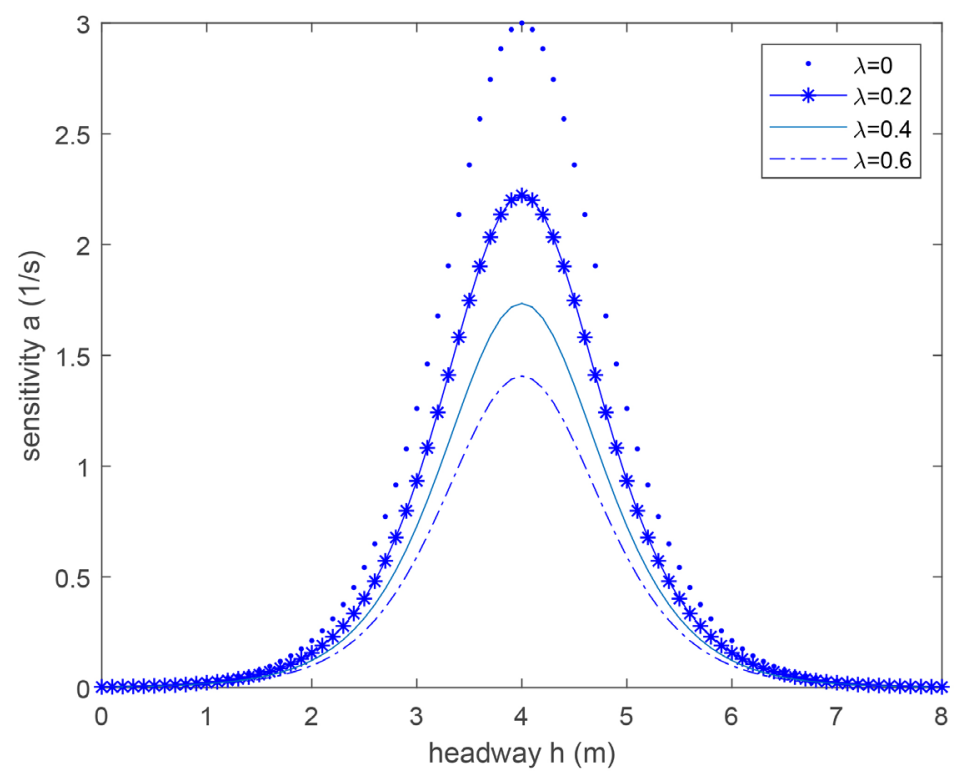

Figure 1. Phase diagram in headway-sensitivity space $(h, a)$.

expansion is the simplest way to describe the behavior of the long-wavelength models.

First, we analyze the slow-varying behavior of long waves near critical point $\left(h_{c}, a_{c}\right)$. Introduce the slow scales $\varepsilon$ of space variable $n$ and time variable $t$ [27], and define the slow variables $X$ and $T$ as follows:

$$
X=\varepsilon(n+b t), \quad T=\varepsilon^{3} t, \quad 0<\varepsilon \ll 1,
$$

where $b$ is a constant. The headway $\Delta x_{n}(t)$ is defined by

$$
\Delta x_{n}(t)=h_{c}+\varepsilon R(X, T) .
$$

Next, by expanding Equation (4) to the fifth-order of $\varepsilon$ with the use of (13) and (14). We obtain the expression:

$$
\begin{aligned}
& \varepsilon^{2} h_{1} \partial_{X} R+\varepsilon^{3} h_{2} \partial_{X}^{2} R+\varepsilon^{4}\left[\partial_{T} R+h_{3} \partial_{X}^{3} R+h_{4} \partial_{X} R^{3}\right] \\
& +\varepsilon^{5}\left[h_{5} \partial_{X T} R+h_{6} \partial_{X}^{4} R+h_{7} \partial_{X}^{2} R^{3}\right]=0 .
\end{aligned}
$$

Here, the coefficients $h_{i}$ are given in Table 1. Where

$V^{\prime}=V^{\prime}\left(h_{c}\right)=\left.\frac{\mathrm{d} V\left(\Delta x_{n}(t)\right)}{\mathrm{d} \Delta x_{n}(t)}\right|_{\Delta x_{n}=h_{c}}$, and $V^{\prime \prime \prime}=V^{\prime \prime \prime}\left(h_{c}\right)=\left.\frac{\mathrm{d}^{3} V\left(\Delta x_{n}(t)\right)}{\mathrm{d} \Delta x_{n}^{3}}\right|_{\Delta x_{n}=h_{c}}$.

Now, we study the traffic flow near critical point $\tau=\left(1+\varepsilon^{2}\right) \tau_{c}$. By taking $b=\frac{V^{\prime}\left(h_{c}\right)}{1+\lambda}$, we eliminate the second- and third-order terms of $\varepsilon$ from Equation (15). The simplified equation of Equation (15) is as follows:

$$
\varepsilon^{4} \partial_{T} R=\varepsilon^{4} m_{1} \partial_{X}^{3} R+\varepsilon^{4} m_{2} \partial_{X} R^{3}-\varepsilon^{3} m_{3} \partial_{X}^{2} R-\varepsilon^{5} m_{4} \partial_{X}^{4} R-\varepsilon^{5} m_{5} \partial_{X}^{2} R^{3} .
$$

Here, the coefficients $m_{i}$ are given in Table 2 .

By transforming variables $X$ and $T$ into variables $x=\varepsilon^{-1} X$ and $t=\varepsilon^{-3} T$, and taking $S(x, t)=\varepsilon R(X, T)$, Equation (16) can be rewritten as follows: 
Table 1. The coefficients $h_{i}$ of (15).

\begin{tabular}{|c|c|c|c|}
\hline$h_{1}$ & $h_{2}$ & $h_{3}$ & \\
\hline$V^{\prime}$ & $(3+\lambda) b^{2} \tau-V^{\prime}$ & $(7+\lambda) b^{3} \tau^{3}-V^{\prime}$ & \\
\hline$-\overline{1+\lambda}$ & $2(1+\lambda)$ & $6(1+\lambda)$ & \\
\hline$h_{4}$ & $h_{5}$ & $h_{6}$ & $h_{7}$ \\
\hline$V^{\prime \prime \prime}$ & 1 & $(15+\lambda) \tau^{3} b^{4}-V^{\prime}$ & $V^{\prime \prime \prime}$ \\
\hline $6(1+\lambda)$ & $\overline{1+\lambda}(3+\lambda) \tau D$ & $24(1+\lambda)$ & $\overline{12(1+\lambda)}$ \\
\hline
\end{tabular}

Table 2. The coefficients $m_{i}$ of (16).

\begin{tabular}{ccc}
\hline$m_{1}$ & $m_{2}$ & $m_{3}$ \\
\hline$\frac{7+\lambda}{6(3+\lambda)^{2}} V^{\prime}-\frac{V^{\prime}}{6(1+\lambda)}$ & $-\frac{V^{\prime \prime \prime}}{6(1+\lambda)}$ & $\frac{(3+\lambda) V^{\prime 2} \tau}{2(1+\lambda)^{3}-\frac{V^{\prime}}{2(1+\lambda)}}$ \\
\hline$m_{4}$ & $m_{5}$ \\
\hline$\frac{(15+\lambda)(1+\lambda) V^{\prime}}{24(3+\lambda)^{3}}-\frac{V^{\prime}}{24(1+\lambda)}-\frac{(7+\lambda) V^{\prime}}{6(3+\lambda)^{2}}+\frac{V^{\prime}}{6(1+\lambda)}$ & $\frac{V^{\prime \prime \prime}}{12(1+\lambda)}$ \\
\hline
\end{tabular}

$$
\partial_{t} S=m_{1} \partial^{3} x S+m_{2} \partial_{x} S^{3}\left(\frac{(3+\lambda) \tau V^{\prime}}{2(1+\lambda)^{3}}-\frac{1}{2(1+\lambda)}\right) V^{\prime} \partial_{x}^{2} S-m_{4} \partial_{x}^{4} S-m_{5} \partial_{x}^{2} S^{3}
$$

By adding term $V^{\prime}\left[\frac{(3+\lambda) \tau V^{\prime}}{(1+\lambda)^{3}}-\frac{1}{1+\lambda}\right] \partial_{x} S$ to both of the left-and right-hand sides of (17) and transforming $t_{1}=t$ and

$$
\begin{aligned}
x_{1}=x-V^{\prime}(1+\lambda)\left[\frac{(3+\lambda) \tau V^{\prime}}{(1+\lambda)^{3}}-\frac{1}{1+\lambda}\right] t \text { in (17), we obtain } \\
\partial_{t_{1}} S=\left(\partial_{x_{1}}-\frac{1}{2} \partial_{x_{1}}^{2}\right)\left\{\left[\frac{7+\lambda}{6(3+\lambda)^{2}}-\frac{1}{6(1+\lambda)}\right] V^{\prime} \partial_{x_{1}}^{2} S\right. \\
\left.+V^{\prime}\left[\frac{(3+\lambda) \tau V^{\prime}}{(1+\lambda)^{3}}-\frac{1}{1+\lambda}\right] S-\frac{\left|V^{\prime \prime \prime}\right|}{6(1+\lambda)} S^{3}\right\} .
\end{aligned}
$$

We define the thermodynamic potentials:

$$
\phi(S) \equiv-\frac{1}{2} V^{\prime}\left[\frac{(3+\lambda) \tau V^{\prime}}{(1+\lambda)^{3}}-\frac{1}{1+\lambda}\right] S^{2}+\frac{\left|V^{\prime \prime \prime}\right|}{24(1+\lambda)} S^{4} .
$$

By taking (19) into Equation (18), the TDGL equation becomes

$$
\partial_{t_{1}} S=-\left(\partial_{x_{1}}-\frac{1}{2} \partial_{x_{1}}^{2}\right) \frac{\delta \Phi(S)}{\delta S}
$$

with

$$
\Phi(S) \equiv \int \mathrm{d} x_{1}\left[\frac{1}{2}\left(\frac{7+\lambda}{6(3+\lambda)^{2}}-\frac{1}{6(1+\lambda)}\right) V^{\prime}\left(\partial_{x_{1}} S\right)^{2}+\phi(S)\right],
$$


where $\phi(S)$ is given by Equation (19), and $\delta \Phi(S) / \delta(S)$ is the derivative of function. The TDGL Equation (20) has two steady-state solutions except for a trivial solution $S=0$. One is the uniform solution

$$
S\left(x_{1}, t_{1}\right)= \pm\left[\frac{6 V^{\prime}\left((3+\lambda) \tau V^{\prime}-(1+\lambda)^{2}\right)}{(1+\lambda)^{2} V^{\prime \prime \prime}}\right]^{\frac{1}{2}},
$$

and the other is the kink solution

$$
\begin{aligned}
S\left(x_{1}, t_{1}\right)= & \pm\left[\frac{6 V^{\prime}\left((3+\lambda) \tau V^{\prime}-(1+\lambda)^{2}\right)}{(1+\lambda)^{2} V^{\prime \prime \prime}}\right]^{\frac{1}{2}} \\
& \times \tanh \left\{\left[3 \times\left(-\frac{(3+\lambda) \tau V^{\prime}}{(1+\lambda)^{2}}+1\right)\right]^{\frac{1}{2}} \times\left(x_{1}-x_{0}\right)\right\},
\end{aligned}
$$

where $x_{0}$ is a constant. Equation (23) represents the coexisting phase.

By the condition

$$
\partial \phi / \partial S=0, \quad \partial^{2} \phi / \partial S^{2}>0 .
$$

Hence, substituting Equation (19) into Equation (24), we can obtain the coexisting curve related to the original parameters

$$
(\Delta x)_{c o}=h_{c} \pm\left[\frac{6 V^{\prime}\left((3+\lambda) \tau V^{\prime}-(1+\lambda)^{2}\right)}{(1+\lambda)^{2} V^{\prime \prime \prime}}\right]^{\frac{1}{2}} .
$$

The spinodal line is given by the following condition

$$
\partial^{2} \phi / \partial S^{2}=0 .
$$

From Equation (18), we obtain the spinodal line described by the following equation

$$
(\Delta x)_{s p}=h_{c} \pm\left[\frac{2 V^{\prime}\left((3+\lambda) \tau V^{\prime}-(1+\lambda)^{2}\right)}{(1+\lambda)^{2} V^{\prime \prime \prime}}\right]^{\frac{1}{2}} .
$$

The critical point is given by the condition

$$
\partial \phi / \partial S=0, \quad \partial^{2} \phi / \partial S^{2}=0 .
$$

Substituting Equation (19) into Equation (28), we have the critical point related to the original parameters as follows:

$$
(\Delta x)_{c}=h_{c}, \quad \tau_{c}=\frac{(1+\lambda)^{2}}{(3+\lambda) V^{\prime}} .
$$

\section{5. mKdV Equation and Its Connection to TDGL}

In this section, based on the stability condition (11) in Section 3, we use the reductive perturbation method to derive the $\mathrm{mKdV}$ equation for the model (4) in 
the unstable region of traffic flow. Similar to the derivation of the TDGL equation in Section 4, we study the slowly varying behavior at long wavelengths near the critical point. We also give slow scales for space variable $n$ and time variable $t$. By inserting $\tau_{c}=\frac{(1+\lambda)^{2}}{(3+\lambda) V^{\prime}\left(h_{c}\right)}$ and $\tau=\left(1+\varepsilon^{2}\right) \tau_{c}$ into Equation (15), making $b=V^{\prime}\left(h_{c}\right) /(1+\lambda)$, and near the critical point, we obtain:

$$
\varepsilon^{4}\left[\partial_{T} R-k_{1} \partial_{X}^{3} R+k_{2} \partial_{X} R^{3}\right]+\varepsilon^{5}\left[k_{3} \partial_{X}^{2} R+k_{4} \partial_{X}^{4} R+k_{5} \partial_{X}^{2} R^{3}\right]=0,
$$

Here, the coefficients $k_{i}$ are given in Table 3.

In Table 3, $V^{\prime}=\mathrm{d} V\left(\Delta x_{n}\right) /\left.\mathrm{d} \Delta x_{n}\right|_{\Delta x_{n}=h_{c}}, V^{\prime \prime \prime}=\mathrm{d}^{3} V\left(\Delta x_{n}\right) /\left.\mathrm{d} \Delta x_{n}^{3}\right|_{\Delta x_{n}=h_{c}}$. We make the following transformation to Equation (30):

$$
T=\frac{1}{k_{1}} T^{\prime}, \quad R=\sqrt{\frac{k_{1}}{k_{2}}} R^{\prime},
$$

so the standard $\mathrm{mKdV}$ equation as follows:

$$
\partial_{T^{\prime}} R=\partial_{X}^{3} R^{\prime}-\partial_{X} R^{\prime 3}-\varepsilon\left[\frac{k_{3}}{k_{1}} \partial_{X}^{2} R^{\prime}+\frac{k_{4}}{k_{1}} \partial_{X}^{4} R^{\prime}+\frac{k_{5}}{k_{2}} \partial_{X}^{2} R^{\prime 3}\right] .
$$

If we ignore the term of $O(\varepsilon)$, Equation (32) is the modified $\mathrm{KdV}$ equation. Its kink solution is given as

$$
R_{0}^{\prime}\left(X, T^{\prime}\right)=\sqrt{c} \tanh \sqrt{\frac{c}{2}}\left(X-c T^{\prime}\right) .
$$

Now, assuming that $R^{\prime}\left(X, T^{\prime}\right)=R_{0}^{\prime}\left(X, T^{\prime}\right)+\varepsilon R_{1}^{\prime}\left(X, T^{\prime}\right)$, we take into account the $O(\varepsilon)$ correction. For the purpose of determining the selected value of the velocity $c$ for the kink solution, it is necessary to satisfy the solvability condition as $\left(R_{0}^{\prime}, M\left[R_{0}^{\prime}\right]\right) \equiv \int_{-\infty}^{+\infty} \mathrm{d} X R_{0}^{\prime} M\left[R_{0}^{\prime}\right]$, where $M\left[R_{0}^{\prime}\right]=\frac{k_{3}}{k_{1}} \partial_{X}^{2} R^{\prime}+\frac{k_{4}}{K_{1}} \partial_{X}^{4} R^{\prime}+\frac{k_{5}}{k_{2}} \partial_{X}^{2} R^{\prime 3}$.

We get the general velocity $c[28]$,

$$
c=\frac{5 k_{2} k_{3}}{2 k_{2} k_{4}-3 k_{1} k_{5}} .
$$

Therefore, the general kink-antikink soliton solution is obtained as follows:

$$
\Delta x_{n}(t)=h_{c} \pm \sqrt{\frac{k_{1} c}{k_{2}}\left(\frac{\tau}{\tau_{c}}-1\right)} \times \tanh \sqrt{\frac{c}{2}\left(\frac{\tau}{\tau_{c}}-1\right)} \times\left[n+\left(1-c k_{1}\left(\frac{\tau}{\tau_{c}}-1\right)\right) t\right] .
$$

Table 3. The coefficients $k_{i}$ of (30).

\begin{tabular}{ccc}
\hline$k_{1}$ & $k_{2}$ & $k_{3}$ \\
\hline$\frac{1}{6(1+\lambda)} V^{\prime}-\frac{(7+\lambda) \tau_{c}^{2}}{6(1+\lambda)^{4}}\left(V^{\prime}\right)^{3}$ & $-\frac{V^{\prime \prime \prime}}{6(1+\lambda)}$ & $\frac{1}{2(1+\lambda)} V^{\prime}$ \\
\hline$k_{4}$ & $k_{5}$ & \\
$\frac{(15+\lambda) \tau_{c}^{3}}{24(1+\lambda)^{5}}\left(V^{\prime}\right)^{4}-\frac{1}{24(1+\lambda)} V^{\prime}$ & $\frac{V^{\prime \prime \prime}}{12} \frac{2(3+\lambda) \tau_{c}}{(1+\lambda)^{3}} V^{\prime}-\frac{1}{1+\lambda}$ &
\end{tabular}


Since the kink soliton solution represents the coexisting phase in the space $(h, a)$, and the kink solution (35) is agreed with the solution (23) obtained from the TDGL Equation (20). We see that the jamming transition can be described by both the TDGL equation with a nontravelling solution and the mKdV equation with a propagating solution [29].

\section{Conclusion}

We propose an extended optimal velocity model (2) by considering the speed limit with intelligent prompts. Using the linear stability theory, the neutral stability line and the critical point of the new model are derived. The stable condition (11) shows that the effect of intelligent prompt limiting speed has a positive effect on expanding the stable area of traffic flow and easing traffic congestion. In addition, the TDGL equation is derived to describe the traffic behavior near the model critical point by using the reduced perturbation method, and the corresponding two steady-state solutions are obtained. From the TDGL, the spinor line and the critical point equation are calculated. At the same time, we derive the $\mathrm{mKdV}$ equation in the unstable region and obtain the relation between TDGL and $\mathrm{mKdV}$ equation.

\section{Acknowledgements}

We thank the Editor and the referee for their comments. This study was supported by the National Natural Science Foundation of China (Grant No.11162020).

\section{Conflicts of Interest}

The authors declare no conflicts of interest regarding the publication of this paper.

\section{References}

[1] Reuschel, A. (1950) Fahrzeugbe wegungen in der kolonne. Oesterreichisches Ingenieur-Archiv, 4, 193-215.

[2] Pipes, L.A. (1953) An Operational Analysis of Traffic Dynamics. Journal of Applied Physics, 24, 274-281. https://doi.org/10.1063/1.1721265

[3] Newell, G.F. (1961) Nonlinear Effects in the Dynamics of Car-Following. Operations Research, 9, 209-229. https://doi.org/10.1287/opre.9.2.209

[4] Bando, M., Hasebe, K., Nakayama, A., Shibata, A. and Sugiyama, Y. (1995) Dynamical Model of Traffic Congestion and Numerical Simulation. Physical Review E, 51, 1035-1042. https://doi.org/10.1103/PhysRevE.51.1035

[5] Helbing, D. and Tilch, B. (1998) Generalized Force Model of Traffic Dynamics. Physical Review E, 58, 133-138. https://doi.org/10.1103/PhysRevE.58.133

[6] Jiang, R., Wu, Q.S. and Zhu, Z.J. (2001) Full Velocity Difference Model for a Car-Following Theory. Physical Review E, 64, Article ID: 017101. https://doi.org/10.1103/PhysRevE.64.017101

[7] Ge, H.X., Cheng, R.J. and Li, Z.P. (2008) Two Velocity Difference Model for a Car Following Theory. Physica A, 387, 5239-5245. https://doi.org/10.1016/j.physa.2008.02.081 
[8] Yuan, N. and Hua, C.C. (2012) Stability and Solitary Wave of Vehicle Following Model with Multiple Front Vehicle Speed Difference. Acta Physica Sinica, 61, Article ID: 160509.

[9] Ge, H.X., Meng, X.P., Zhu, K.Q. and Cheng, R.J. (2014) The Stability Analysis for an Extended Car Following Model Based on Control Theory. Chinese Physics Letters, 31, Article ID: 080505. https://doi.org/10.1088/0256-307X/31/8/080505

[10] Zhou, T., Sun, D.H., Li, H.M. and Liu, W.N. (2014) A New Coupled Map Car-Following Model Considering Driver's Steady Desired Speed. Chinese Physics B, 23, Article ID: 050203. https://doi.org/10.1088/1674-1056/23/5/050203

[11] Zhu, W.X. and Zhang, L.D. (2018) A New Car-Following Model for Autonomous Vehicles Flow with Mean Expected Velocity Field. Physica A, 492, 2154-2165. https://doi.org/10.1016/j.physa.2017.11.133

[12] Zhai, C. and Wu, W.T. (2018) A New Car-Following Model Considering Driver's Characteristics and Traffic Jerk. Nonlinear Dynamics, 93, 2185-2199. https://doi.org/10.1007/s11071-018-4318-7

[13] Nagatani, T. (1998) Thermodynamic Theory for the Jamming Transition in Traffic Flow. Physical Review E, 58, 4271. https://doi.org/10.1103/PhysRevE.58.4271

[14] Nagatani, T. (1999) TDGL and MKdV Equations for Jamming Transition in the Lattice Models of Traffic. Physica A, 264, 581-592. https://doi.org/10.1016/S0378-4371(98)00466-X

[15] Nagatani, T. (1998) Modified KdV Equation for Jamming Transition in the Continuum Models of Traffic. Physica A, 261, 599-607. https://doi.org/10.1016/S0378-4371(98)00347-1

[16] Lv, F., Zhu, H.B. and Ge, H.X. (2014) TDGL and mKdV Equations for Car-Following Model Considering Driver's Anticipation. Nonlinear Dynamics, 77, 1245-1250. https://doi.org/10.1007/s11071-014-1374-5

[17] Jin, Z.Z., Li, Z.P., Cheng, R.J. and Ge, H.X. (2018) Nonlinear Density Wave Investigation for an Extended Car-Following Model Considering Driver's Memory and Jerk. Modern Physics Letters B, 32, Article ID: 1750366.

https://doi.org/10.1142/S0217984917503663

[18] Song, H., Zheng, P.J. and Ge, H.X. (2017) TDGL and mKdV Equations for an Extended Car-Following Model. Nonlinear Dynamics, 90, 2253-2262. https://doi.org/10.1007/s11071-017-3747-z

[19] Jin, Z.-Z., Li, Z.P., Cheng, R.J. and Ge, H.X. (2018) Nonlinear Analysis for an Improved Car-Following Model Account for the Optimal Velocity Changes with Memory. Physica A, 501, 278-288. https://doi.org/10.1016/j.physa.2018.05.043

[20] Li, Z.P. and Liu, Y.C. (2006) Analysis of Stability and Density Waves of Traffic Flow Model in an ITS Environment. The European Physical Journal B, 53, 374-376. https://doi.org/10.1140/epjb/e2006-00382-7

[21] Xie, D.F., Yao, Z.Y. and Zhao, X.M. (2008) The Effect of ACC Vehicles to Mixed Traffic Flow Consisting of Manual and ACC Vehicles. Chinese Physics B, 17, 1674. https://doi.org/10.1088/1674-1056/17/12/018

[22] Yao, J., Huang, J.Y., Chen, G.R. and Xu, W.S. (2013) A New Coupled-Map Car-Following Model Based on a Transportation Supernetwork Framework. Chinese Physics B, 22, Article ID: 060208. https://doi.org/10.1088/1674-1056/22/6/060208

[23] Li, Z.P., Li, W.Z., Xu, S.Z. and Qian, Y.Q. (2015) Analises of Vehicle's Self-Stabilizing Effect in an Extended Optimal Velocity Model by Utilizing Historical Velocity in an Environment of Intelligent Transportation System. Nonlinear 
Dynamics, 80, 529-540. https://doi.org/10.1007/s11071-014-1886-z

[24] Kuang, H., Xu, Z.P., Li, X.L. and Lo, S.M. (2017) An Extended Car-Following Model Accounting for the Average Headway Effect in Intelligent Transportation System. Physica A, 471, 778-787. https://doi.org/10.1016/j.physa.2016.12.022

[25] Zhang, G., Sun, D.H., Liu, W.N. and Liu, H. (2015) Traffic Stability of a Car-Following Model Considering Driver's Desired Velocity. Modern Physics Letters B, 29, Article ID: 1550097. https://doi.org/10.1142/S0217984915500979

[26] Wu, S.Z., Cheng, R.J. and Ge, H.X. (2011) The Time-Dependent Ginzburg-Landau Equation for the Two-Velocity Difference Model. Chinese Physics B, 20, Article ID: 080509. https://doi.org/10.1088/1674-1056/20/8/080509

[27] Cross, M.C. and Hohenberg, P.C. (1993) Pattern Formation Outside of Equilibrium. Reviews of Modern Physics, 65, 851. https://doi.org/10.1103/RevModPhys.65.851

[28] Ge, H.X., Cheng, R.J. and Dai, S.Q. (2005) KdV and Kink-Antikink Solitons in Car-Following Models. Physica A, 357, 466-476. https://doi.org/10.1016/j.physa.2005.03.059

[29] Nagatani, T. (1998) Time-Dependent Ginzburg-Landau Equation for the Jamming Transition in Traffic Flow. Physica A, 258, 237-242.

https://doi.org/10.1016/S0378-4371(98)00211-8 